\title{
Reliability Analysis Algorithm for Multiple Microgrids in Distribution Systems Based on Complex Network Mathematical Theory
}

\author{
FABIAN RODRIGUEZ, SERGIO RIVERA* \\ Electrical and Electronics Engineering Department \\ Universidad Nacional de Colombia \\ Carrera 30 Número 45-03, Bogotá \\ COLOMBIA
}

\begin{abstract}
It exists a great potential in microgrids connected to distribution systems of being taken into advantage reconfiguration possibilities with the purpose of achieving the quality of service regulatory requirements that become more demanding each day. In addition, it is possible to optimize the network operators' income by increasing the incentives for upgrading the quality indexes. In this paper, it's proposed an evaluation algorithm of the connection points of multiple microgrids in a distribution system that upgrades the reliability of the system as a whole, being based in complex network analysis (CNA), a perspective of power systems that allows the evaluation of an electrical system as a graph. For that, a model of a trial system is made from the CNA point of view utilizing the MATLAB software and afterwards, as validation of the proposal of this work, the system's reliability is evaluated by connecting multiple microgrids into critical nodes provided by the CNA making use of the NEPLAN tool of power systems simulation.
\end{abstract}

Key-Words: - Complex network mathematical theory; operation in uncertain environments; optimization; reliability; smart microgrid

Received: March 15, 2021. Revised: December 23, 2021. Accepted: January 20, 2022. Published: February 11, 2022.

\section{Introduction}

Historically, the importance of the reliability analysis of the performance of power systems is proportional to the tension level of the operation and the main focus was in the generation stage and in the energy transmission. However, approximately $80 \%$ of all the interruptions that the clients suffer occur due to failures in the distribution networks [1]. Due to the above, the performance evaluation in respect to the reliability (or quality of the technical service) of distribution systems has been widely studied since the decade of 1930 as a key aspect in the planning of electrical energy systems [2], and since then countless studies that develop new techniques, models and reliability analyses applications in these systems have been published [2], [3].

Despite the great quantity of studies and publications and the progress made in new analysis techniques thanks to the increasing development of computing tools that allow advanced analyses to be made, studies remain to be developed and investigation is still being made in this field, focusing in networks with high penetration of DER (Distributed Energy Systems) [4], NCRES (Non-
Conventional Renewable Energy Sources) [5], microgrids and in the management of assets and maintenance [6]-[11]. So, it's evident that the reliability analyses still hold their importance in the planning of power systems and distribution networks.

Taking into account that in the past years the regulation of the Colombian electrical sector has implemented different mechanisms to promote and incentivize the connection of "small scale auto generators (SSAG)", "NCRES (Non-Conventional Renewable Energy Sources)" and "DER (Distributed Energy Systems)" in the "National Interconnected System (NIS)" and in the "Not Interconnected Zones (NIZ)", through different norms [12]-[15].

The microgrids have the capacity of improving the tension regulation, the energy quality, the protection schemes, diminish losses from the system and the emissions due to the nature of the used technologies and substantially improve the system's reliability thanks to its reconfiguration capability before events and to operate isolated from the network [7], [9], [16]. In such virtue, it exists a great 
potential in taking advantage of the microgrids to achieve the quality of service regulatory requirements and also optimize the network operators' incomes by increasing the incentives for the improvement of quality indexes.

The reliability analyses are based mainly in two aspects: the individual parameters of each one of the elements that conform the electrical system such as the failure rate, availability, repair time, average failure time, average time between failures, etc., and in how every one of these elements are connected, that is to say, the topology of the network. However, due to the complexity of the future energy distribution networks, it exists an urgent necessity of upgrading these traditional reliability evaluation methods into new techniques [7].

One of the new tools for the electrical systems analysis that has been growing in interest is the complex network theory [17]. Taking the above into account, the complex network theory is shown as a new tool that can assist not only the efficient processing in the calculation, but also in giving a new perspective in the problem's analysis. Some of the studies developed from the point of view of the complex networks and focused in the power system's analysis are the following:

In [18] a vulnerability and recognition analysis of key nodes in the electrical networks from the complex networks' perspective is made. Using the centrality of the nodes from the complex network theory, the key nodes are identified and the system's behavior is analyzed by modifying the network's parameters to correct the centrality of the vulnerable nodes. Indicators are introduced as the netability and the vulnerability index to describe the transfer and performance capacity in a normal functioning and to evaluate the vulnerability of the electrical system in case of waterfalling failures.

In [19] it's studied how a waterfalling failure in electrical networks is produced and the correlation between the key parameters is searched using the complex network theory to improve the sturdiness of electrical networks. In [20] a methodology for evaluating the stability of a Smartgrid that includes microgrids is proposed. For this evaluation an index called Intermediation Index that is based in the theory of complex networks is taken. An improved intermediation index is proposed, since it's considered the real charge flow through the transmission lines along the network. This work is a starting point in the investigation area of complex systems to evaluate the stability of power systems.

In [21] an indicator that is based in the impedance of the transmission lines as a criterion to measure the vulnerability of the system is proposed. This indicator, obtained through the analysis of complex networks, can identify the critical transmission lines of the network, whether by its position in the system or by the power transmitted along the network.

In the work developed in [22] a systematic method based in the complex network theory is stablished to propose that, in normal conditions, every modern system of distributed generation with variable topology and bounded control entries, can be represented as a Hamiltonian stable system. With the prior premise, it's analyzed a microgrid driver to evaluate the proposed method.

A methodology for the optimal localization of microgrids in electrical distribution systems using complex network analysis [23]. The optimal localization in this work is acknowledged as the localization that gives as a result a greater resilience in the network, a reduction in the energy losses an in the lines' chargeability, a better voltage stability and the supply to the critical charges during a blackout [24]-[32]. The criteria used to select the optimal localization of the microgrids were based in the centrality analysis taken from the complex network theory [33]-[41].

The work in [42] showed that the network's structure influences in a very important way the reliability of the system. Additionally, in the same article it was observed that the reliability of the microgrids is very sensible to many other factors; such as the system's demand, the network structure and the coupling method proposed. The literature revision [7], [43], [44] shows that there are many factors that must be taken into consideration to determine in an exhaustive way the reliability of the microgrids. Some of the factors include the type of failure, the weather and the network's structure.

Taking into account that the microgrids have the property of improving the reliability of the distribution networks, as evaluated in [9], where the SAIDI and SAIFI indicators were used for the evaluation, it exists a great potential for the microgrids to be taken into advantage to fulfill the quality of service regulatory requirements and to optimize the income of the network operators by increasing the incentives for improving the quality indexes.

In this way, by taking into account that the reliability analysis is based fundamentally in the topology of the network, in this work is proposed the use of complex networks to analyze, from other perspective, the topological parameters of the network that allow the identification of nodes in which the microgrids connection achieves an improvement in the general reliability of the system. 


\section{Materials and Methods: Complex Network Analysis in Electrical Systems}

In the formality of network analysis, a network is a group of elements called vertexes or nodes, with different interconnections between them, (these connections can be power lines or also communication interconnections), called edges. The systems that take the form of networks (or graphs) are plenty in the world. The examples include: internet, social networks, organizational networks, networks for commercial relationships between companies, public transportation networks, and for our case, electrical distribution networks, and many others [45], [46].

The different fields' researchers have stablished, along the past years, a wide array of mathematical, computing and probabilistic tools, destined to study, model, and comprehend different network systems. The study of the network science stablished its basic foundations in the development of the graph theory, that was explored for the first time by Leonhard Euler in 1736, when he published the article Seven Bridges of Königsberg [47]. The solution to the problem of the seven bridges of Königsberg is generally considered as a starting point for the graph theory and the network science [46], [48].

\subsection{Complex Networks}

The complex network theory is a new discipline that has as main focus to analyze different static topological characteristics, as well as dynamic behaviors in interconnected systems at great scale. The theoretical work in complex networks came to be from the graph theory and the network science [45], [46].

In the context of the network theory, a complex network could be defined as a graph that is made up from many nodes related between them [49]. It could also be defined as a network that has nonobservable topological characteristics that don't surface in simple networks such as random ones, but that often occur in real systems' graph models [50].

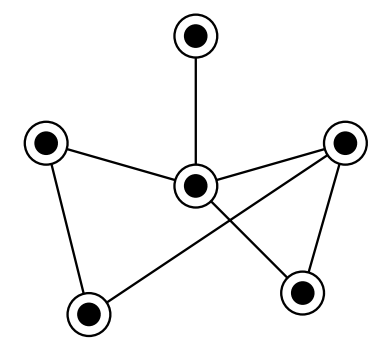

(a)
Without taking into account the intrinsic dynamic of every component, a complex network can be described simply as a graph or a $\mathrm{G}$ network, that can be defined as a finite group of ordered pairs $G=(V$, $\mathrm{E}$ ), where $\mathrm{V}$ is a subgroup of non-empty elements called nodes or vertexes and $\mathrm{E}$ a subgroup formed by ordered pairs of elements different from $\mathrm{V}$, called borders or edges [51].

In Figure 1 ([51]) a non-directed graph is exemplified, $\mathrm{G}=(\mathrm{V}, \mathrm{E})$, where $\mathrm{V}=\{\mathrm{v} 1, \mathrm{v} 2, \ldots, \mathrm{v} 5\}$ and $\mathrm{E}=\{\mathrm{v} 1 \mathrm{v} 2, \mathrm{v} 2 \mathrm{v} 3, \mathrm{v} 3 \mathrm{v} 4, \mathrm{v} 3 \mathrm{v} 5, \mathrm{v} 2 \mathrm{v} 5, \mathrm{v} 4 \mathrm{v} 5\}$.

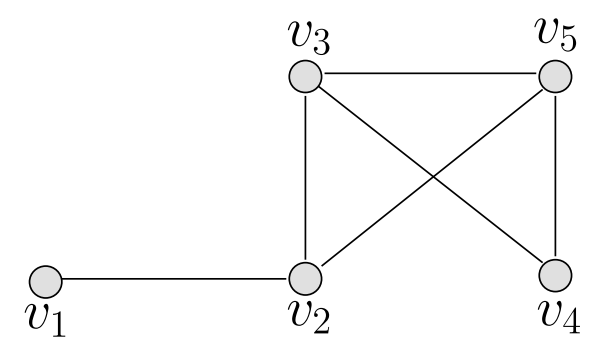

Fig. 1: Example of a 5 vertexes non-directed graph.

A group of vertexes joined together by borders is the most simple of network types; but generally, the real networks are much more complex than this. For example, there can be more than just one different type of vertex in a network, or more than one type of edge. And the vertexes or edges can have a variety of properties, numerical or others, associated to them. A graph is considered directed when a direction is assigned to the edges that form them, like the graph shown in Figure 2(d), in which the directed graph $\mathrm{D}=(\mathrm{V}, \mathrm{E})$ has ordered pairs (vi, $\mathrm{vj}) \in \mathrm{E}$, where vi is the end of the edge and vj the start.

If to every one of the graph's edges is associated a value or w cost, it's resulted in a graph $\mathrm{Gw}=(\mathrm{V}, \mathrm{E}$, w) called graph with weights like the one shown in Figure 2(c). In these graphs, there can be considered short or geodesic paths between vertexes, since the longitude notion is introduced, defined as the sum of the weights along a trajectory between vertexes [46].

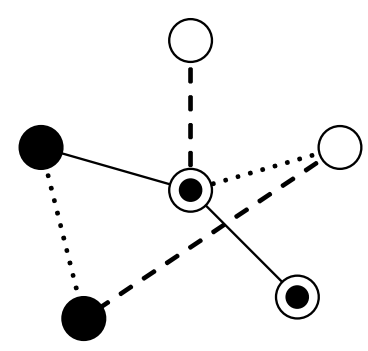

(b) 


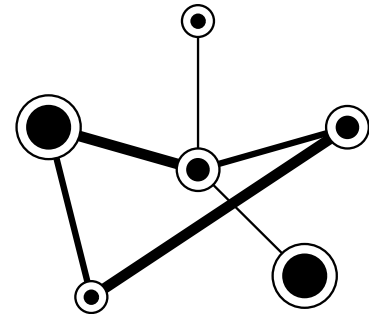

(c)

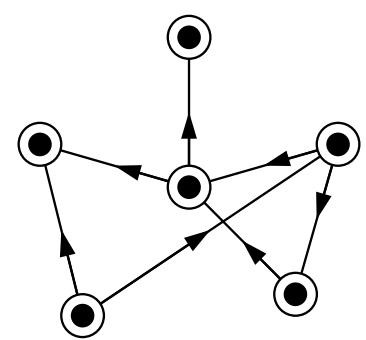

(d)

Fig. 2: Examples of network types (a) non-directed graph with simple vertexes and edges; (b) graph with different types of discrete vertexes and edges; (c) graph with weights of vertexes and edges; (d) directed graph where each edge has a direction.

\subsection{Power Systems from the Point of View of Complex Networks}

A power system is also formed by vertexes (nodes, substations, derivations, barrages) and edges (transmission or distribution lines, power transformers) between them as a complex network [52], thus, the electric energy distribution systems can be qualified as complex networks and be studied through the optic of complex network analysis (CNA) [20].

To study an electrical distribution network using the complex network analysis, the first step is to model the network as a graph. In the context of complex networks and for the present analysis, the electrical nodes, substations, generation centers and microgrid connection points with the distribution system correspond to the vertexes, while the distribution lines and transformers correspond to the edges.

In Figure 3 is shown the original unilinear diagram of the IEEE trial system with 30 nodes that will be used as case of study. This system counts with 30 nodes connected through 41 impedances (lines or transformers).

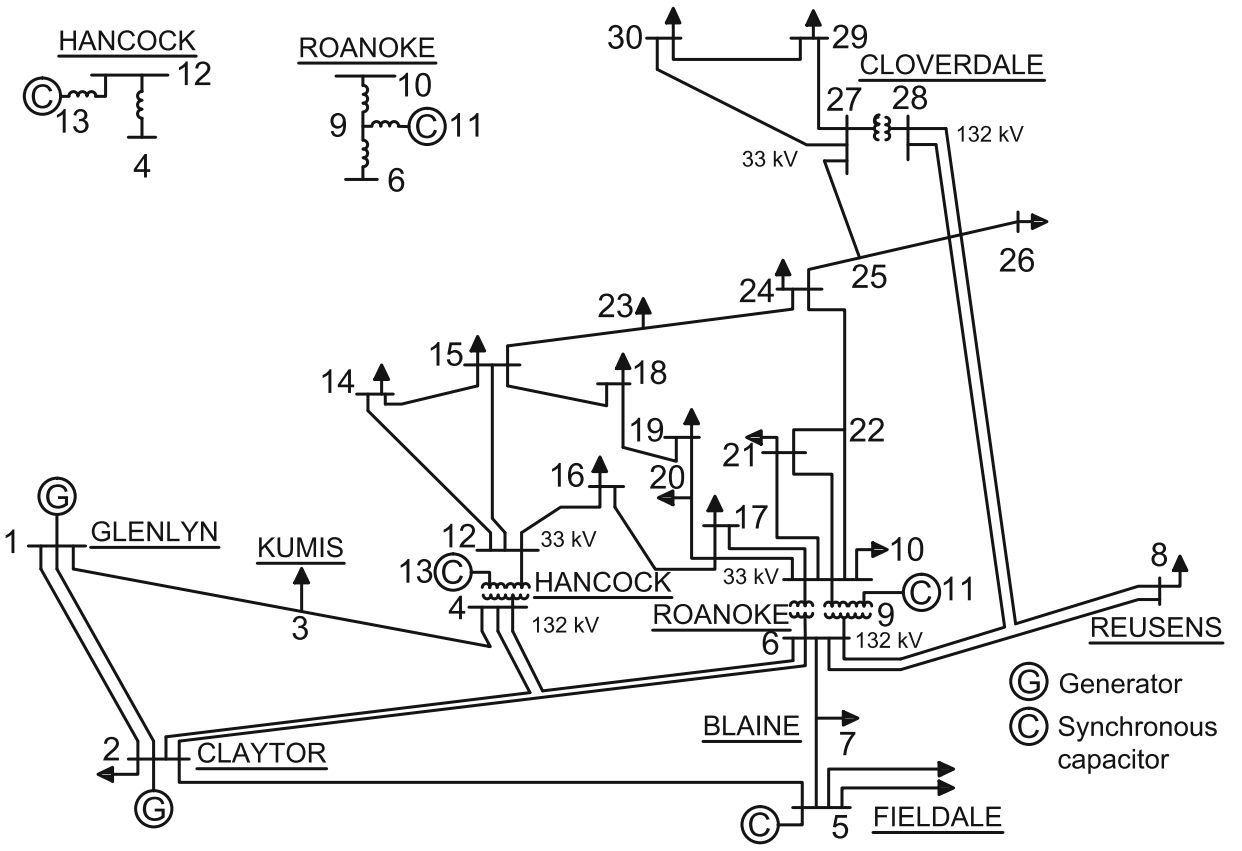

Fig. 3: General unifiliar diagram of the IEEE trial system with 30 nodes. Source: Figure taken from http://labs.ece.uw.edu/pstca/pf30/pg_tca30bus.htm.

In Figure 4 is shown the IEEE system with 30 nodes as a non-directed graph and in Figure 2-5 is shown the topology mapping of the system. The next aspect to take into consideration for the network's analysis as a graph is to formulate the weight matrix of the graph (adjacency matrix with weights). The traditional focus of the complex network analysis only considered the physical connection [17], [53]. This model does not reflect one of the fundamental aspects of the electrical energy system as it is the impedance, that plays an important role in the power flow, the losses and stability of the system. 


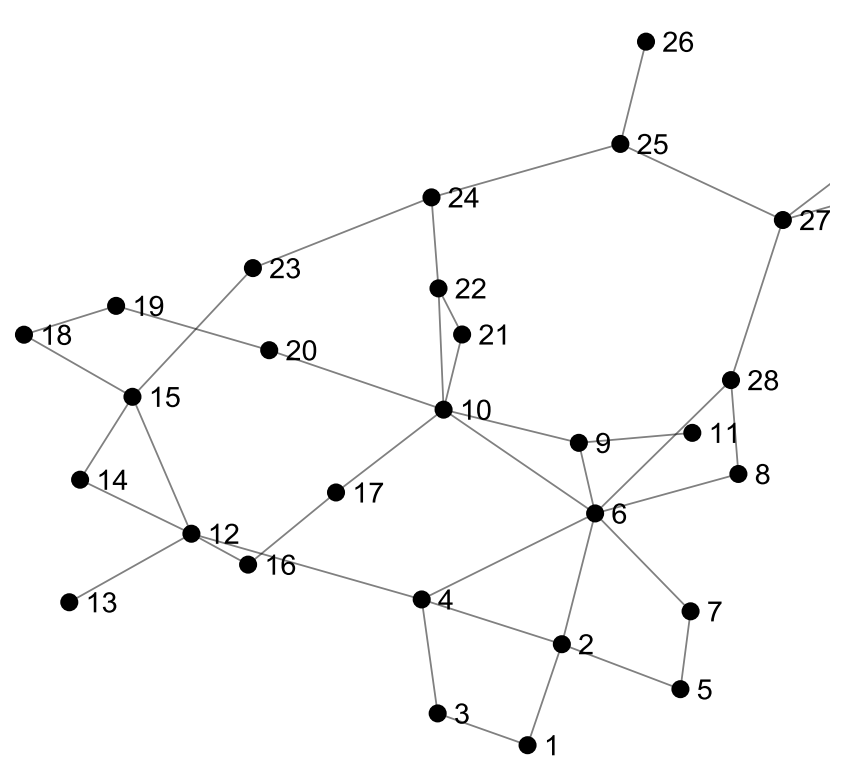

Fig. 4: IEEE system with 30 nodes as a non-directed graph.

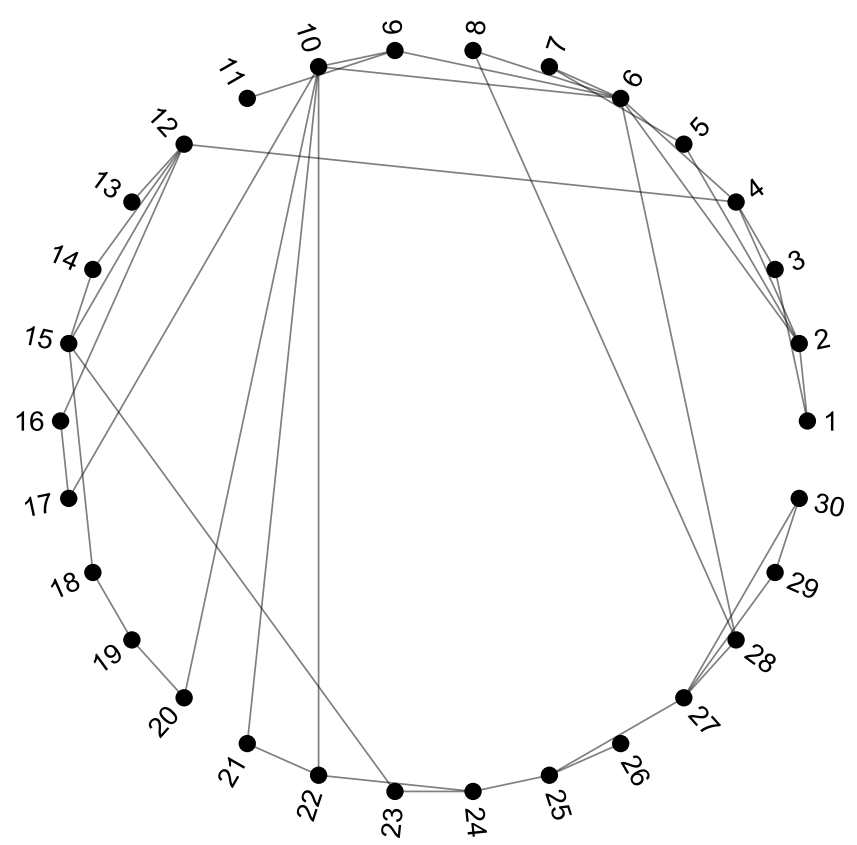

Fig. 5: Topology mapping of the IEEE system with 30 nodes.

The focus to find the adjacency matrix with weights is based in the admittance matrix [54]. In this case, the weight matrix can be found from the elements outside the admittance matrix's diagonal. For a system of $\mathrm{n}$ nodes, the voltage equation is written in matrix form as:

$$
\begin{gathered}
{\left[\begin{array}{c}
I_{1} \\
I_{2} \\
\vdots \\
I_{i} \\
\vdots \\
I_{n}
\end{array}\right]=\left[\begin{array}{cccccc}
Y_{11} & Y_{12} & \cdots & Y_{1 i} & \cdots & Y_{1 n} \\
Y_{21} & Y_{22} & \cdots & Y_{2 i} & \cdots & Y_{2 n} \\
\vdots & \vdots & & \vdots & & \vdots \\
Y_{i 1} & Y_{i 2} & \cdots & Y_{i i} & \cdots & Y_{i n} \\
\vdots & \vdots & & \vdots & & \vdots \\
Y_{n 1} & Y_{n 2} & \cdots & Y_{n i} & \cdots & Y_{n n}
\end{array}\right]\left[\begin{array}{c}
V_{1} \\
V_{2} \\
\vdots \\
V_{i} \\
\vdots \\
V_{n}
\end{array}\right]} \\
\boldsymbol{I}_{\text {bus }}=\boldsymbol{Y}_{\text {bus }} \boldsymbol{V}_{\text {bus }}=
\end{gathered}
$$

Where Ybus is the admittance matrix. The diagonal elements of the admittance matrix correspond to the sum of the impedances of the lines connected to each one of the system's nodes. Given that the diagonal elements are not included in the weight matrix, for this analysis the impedances between the nodes and the ground are not considered. The elements outside the diagonal are equal to the negative of the admittance equivalent between the nodes. So, in this case, the element ij of the weight matrix $[\mathrm{A}(\mathrm{Gw})]$ can be found from wij $=$ Yij.

It's evident that the Ybus matrix is a symmetrical matrix, that is to say that, $\mathrm{Yij}=\mathrm{Yji}$, thus, by being equal the impedance in both ways, the power flow's directionality is not considered in this model and is considered a non-directed graph.

For the system's modelling as a complex network, some considerations like the following are made:

It is assumed that the system is balanced.

The power transformers and transmission lines are modeled as edges with weights, being the weight equal to each one of the components in p.u.'s admittance.

The parallel lines between two substations or nodes are considered as a single equivalent transmission line with the purpose of simplifying the graph.

\subsection{Centrality Measures}

From the complex network analysis perspective numerous measures or indexes that can define certain characteristics in the network knowing the network's structure have been developed. For example, the social scientists have used some centrality indexes to better explain the impact a person has inside of a network [55]-[57]. Between these centrality indexes, the more used in electrical energy systems are the grade centrality and the betweenness centrality.

\subsubsection{Grade Centrality}

The simplest one of the centrality measures is the grade centrality. It's defined as the number of edges possessed by a node that connects it to the others 
[45]. The grade centrality can be obtained from the matrix's grade diagonal. In complex networks, most of the nodes have a low grade, but a hierarchy of higher-grade nodes called "hubs" exists, which have an important role inside the network. In the electrical networks, these hubs correspond to important transformation or generation substations and is called system's hub to the vertex with the highest grade. In the case of the considered 30 node system, the hub node corresponds to the node 7 that has 6 grade and the average system's grade is 2.73 . In Figure 6 is shown the grade distribution for the system under study.

\subsubsection{Betweenness Centrality}

The betweenness centrality could be defined as the number of shortest paths between the vertexes (nodes) that pass through this vertex. The betweenness centrality measures how many times a vertex is found in the direct path between any other pair of vertexes [45].

A high betweenness value indicates that a vertex can reach other vertexes in relatively short trajectories, or that a vertex is found in a considerable fraction of the shortest trajectories that connect pairs of other vertexes. The betweenness can be calculated through the Equation (6).

$$
c(v)=\sum_{s, t \neq u} \frac{n_{s t}(v)}{N_{s t}}
$$

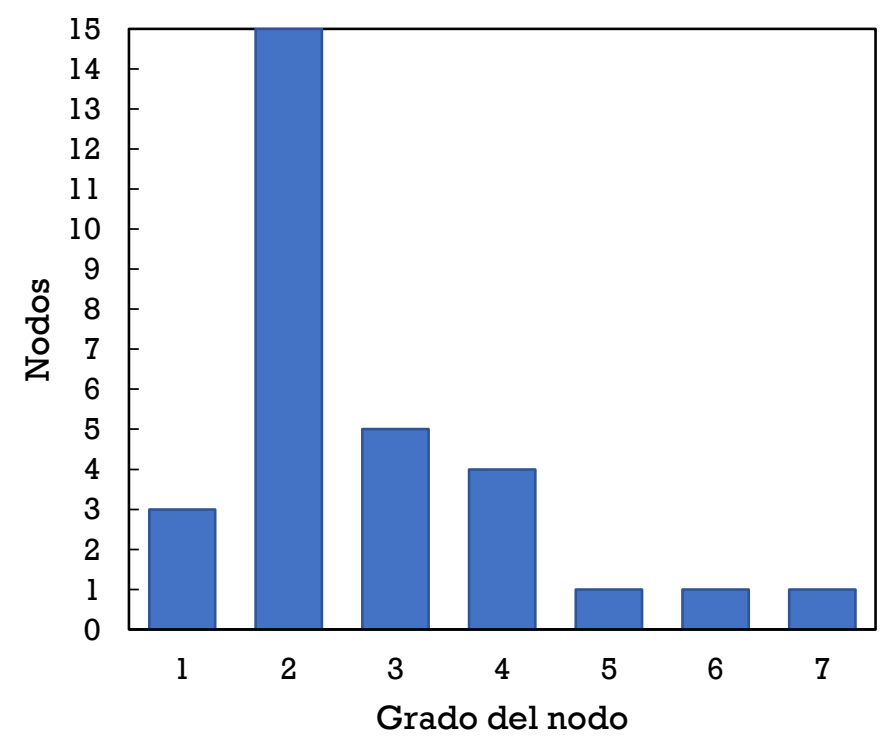

Fig. 6: Grade distribution for the IEEE trial system with 30 nodes.

Where nst(v) is the number of shortest paths between the vertexes $s$ and $t$ that pass through the vertex $\mathrm{v}$, and Nst is the total number of shortest paths between the vertexes $s$ and $t$.

\subsubsection{Closeness Centrality}

The closeness centrality could be defined as the average of shortest routes between a specific node and the rest of the network's nodes. A high value in the closeness centrality of a node indicates how close it is from the other nodes. The shortest electrical route is the one that has the minimum impedance possible, thus, the edges' weights are chosen in function of the impedance. The closeness centrality can be calculated using the Equation (27).

$c(i)=\left(\frac{v_{i}}{N-1}\right)^{2} \frac{1}{C_{i}}$
Where vi is the number of close vertexes from the vertex $\mathrm{i}$ (without including $\mathrm{i}$ ), $\mathrm{N}$ is the number of vertexes in the $\mathrm{G}$ graph and $\mathrm{Ci}$ is the total sum of the distances between the vertex $\mathrm{i}$ to any nearby vertexes. If a path from the vertex $i$ to the nearby vertexes does not exist, then c(i) is zero.

\subsubsection{Clustering}

The clustering is an index that evaluates how interrelated the neighboring nodes inside of a vertex are. The local clustering CC(i) of a node calculates the average connections of its nearby nodes. The $\mathrm{C}(\mathrm{i})$ for each node and the total $\mathrm{CC}(\mathrm{G})$ of the network can be calculated with the equations (2-8) and (2-9), respectively:

$C C(v)=\frac{2 N_{v}}{K_{v}\left(K_{v}-1\right)}$ 


$$
C C(G)=\frac{1}{n} \sum_{i=1}^{n} C_{i}
$$

Where, $\mathrm{Nv}$ is the number of edges between the neighbors of the node $\mathrm{v}$ and $\mathrm{Kv}$ is the node $\mathrm{v}$ 's grade.

From the point of view of electrical systems, a node with a high $\mathrm{CC}(\mathrm{v})$ could indicate that, if separated, the power flow will have alternative routes to the closest nodes.
In other words, a high node coefficient $\mathrm{CC}(\mathrm{v})$ could indicate a less central node. The total $\mathrm{CC}(\mathrm{G})$ of the IEEE system with 30 nodes is 0.2348 . The $\mathrm{CC}(\mathrm{G})$ indicator is equal to 1 in a graph if every vertex in the graph are connected to each other in the same way.

In Table 1 are summarized some of the centrality measures applied in the IEEE trial system with 30 nodes.

Table 1 . Summary of the IEEE network with 30 nodes' measures from the CNA perspective.

\begin{tabular}{|c|c|}
\hline Number of nodes & 30 \\
\hline Edges & 41 \\
\hline Maximum grade & 7 \\
\hline Average grade & 2.73 \\
\hline Clustering & 0.2348 \\
\hline Average betweenness & 33.433 \\
\hline
\end{tabular}

\section{Reliability Analysis of Multiple Microgrids}

\subsection{Case of Study}

Taking as a base the IEEE trial circuit with 30 nodes [58], [59], a case of study was developed making use of the power systems simulation tool NAPLAN. In Figure 6 is shown the unifiliar diagram of the simulated system and the connection points of the microgrids for the based case.

The original IEEE trial model with 30 nodes does not have assigned longitudes for the lines, the characterization of the lines is made only with the total electrical parameters of resistance, reactance and susceptance of each one of the lines. The longitude parameter of the lines is fundamental for the reliability analysis in distribution networks, in a way that it was estimated the longitude taking typical reactance values per unit of longitude with the purpose of assigning the longitude of each one of the model's lines and apply the reliability parameters according with the IEEE 493-2007 norm [60] per unit of longitude for each one of the lines.

For the analysis is considered that all the elements connected through nodes (edges, in this case lines and transformers of the system) count with cut elements and protection in both ends. The charges, capacitances, generators and the microgrids in the connection points with the distribution system also have elements of cut and protection.

The system was modified locating in the node 1 (slack node with a generation unit in the original model) an infinite bar, with the purpose of simulating the connected network to an interconnected network of great capacity with an ideal reliability. The original model's generators of

the IEEE network with 30 nodes were replaced by microgrids to simulate multiple microgrids connected to the system. In a way that the modified system counts with 5 microgrids.

The maximum capacities of the elements were modified so that in the network's initial conditions there aren't any overcharged lines and transformers, also that the tension in every node is between 0.9 and 1.0 p.u., because of the contrary this condition would be taken as a contingency and in the original IEEE model with 30 nodes there is an overcharged line to the charge flow channel. The range criterion of allowable tensions was taken from the Code of Networks in its section Operation Code [61].

The reliability parameters for the elements that form the system, such as the failure rate, repair time, MTTF and MTTR were taken from the IEEE standard 493-2007 [60]. The reliability parameters for the microgrid were taken with a base in the article [62].

It's considered that the microgrids are connected to the distribution system of intermediate tension through an impedance that represents the IC (Interlinking Converter between the MG and the distribution network) in the case of microgrids with DC bus or the coupling transformer in case of microgrids of AC bus.

The relation between the internal generation and the charge (Generation-Load Ratio GLR) of the microgrids is important in the evaluation on the reliability of the distribution system to which they're connected. A microgrid's GLR can be minor, greater or equal to 1 ; it's minor when the microgrid does not satisfy every necessity of the consumer's charge in its interior, although it has the 
capacity to do so (for example, for economical reasons), and it's greater than one when it can export energy to the distribution network [9].

Taking into account then that it's fundamental to considerate the GLR in the evaluation of the reliability of the system, since the backup that can give the microgrid to the system before events of failure from the network's elements depends on it. The microgrids are modeled with the VPP concept (Virtual Power Plant) [63], through a model of distributed generation and a charge and the parameters of these elements are varied with the purpose of simulating the GLR of the microgrid.

The VPP concept is employed as a tool to facilitate the reliability evaluation in the active distribution network with multiple microgrids. The concept consists in considering every one of the microgrid's components in a single entity to offer a simplified equivalent model and to be used in the distribution network. When it's integrated in a distribution network, the functionality of a microgrid is to interchange energy with the distribution network, this way the microgrid becomes an energy source if it outputs more energy than the local charge and it becomes into a charge when the charge exceeds the available output. Thus, in a similar way to a conventional generation plant, a VPP will be represented by a model of multiple states in the reliability analysis [63].
For the analyzed case, the generation capacities of the microgrids are considered equal for all five subsystems and are assumed as 40 MVA (34 MW, 21 MVAr). The microgrid's charge is variable according to the GLR as exposed beforehand. For the present analysis the variable charge will be considered in function to the GLR but with a constant power factor of 0.85 .

The microgrid's contribution to the reliability tends to be greater with higher GLR values. However, the impact that the GLR has in the reliability depends in the microgrid's localization in the system and in the localization of the failure for a specific case [9].

\subsection{System's Reliability Evaluation}

By using as a NEPLAN software as a simulation tool, the SAIDI, SAIFI and CAIDI reliability indexes were calculated for the configuration of the modified IEEE system with 30 nodes that is shown in Figure 6.

With the purpose of verifying the impact that the GLR has in the reliability of the system, the reliability indexes were evaluated for different values of the GLR and one case without including the microgrids in the system. The results are shown in Table 2.

Table 2. Summary of the reliability indicators for different cases with MG for the analyzed IEEE system with 30 nodes.

\begin{tabular}{lllll}
\hline Indicator & Unit & Without MG & $\begin{array}{l}\text { With } \\
\text { GLR=2 }\end{array}$ & $\begin{array}{c}\text { MG,With } \\
\text { GLR }=1.5\end{array}$ \\
\hline $\mathrm{N}$ & Clients & 21 & 26 & 26 \\
\hline SAIFI & 1/yr. & 2.307 & 0.442 & 0.184 \\
\hline SAIDI & min/yr. & 1254 & 320.327 & 243.748 \\
\hline CAIDI & $\mathrm{h}$ & 9.061 & 12.092 & 22.118 \\
\hline $\mathrm{P}$ & $\mathrm{MW} / \mathrm{yr}$. & 750.35 & 149.914 & 211.018 \\
\hline $\mathrm{W}$ & $\mathrm{MWh} / \mathrm{yr} .5250 .5$ & 1640.39 & 2280.141 \\
\hline
\end{tabular}

Note: P corresponds to the total power that was stopped from supply in a year and W corresponds to the total energy that was stopped from supply in a year.

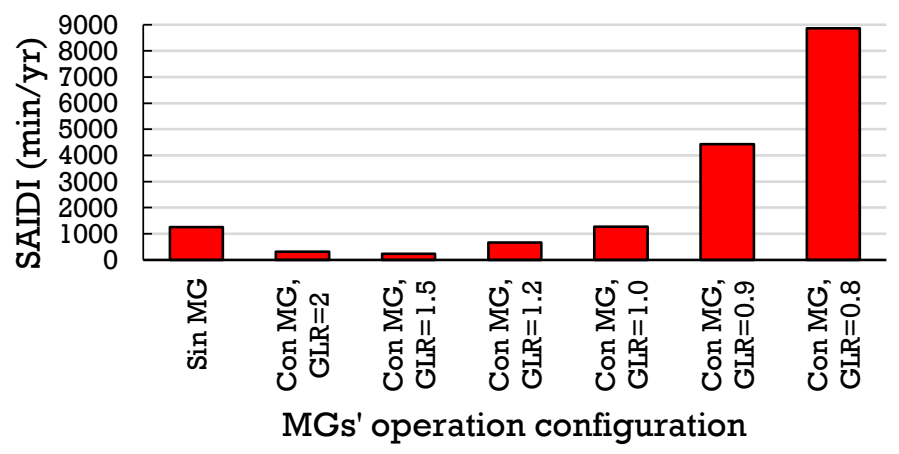

Fig. 7: Behavior of the SAIDI for different values of GLR of the MGs. 
The probability of system's failure grows exponentially by diminishing the GLR of the microgrids. This shows that effectively the backup that the microgrids can give to the system is fundamental in the reliability analysis. It's also evidenced that the system without including the MGs has a similar reliability to the system when the MGs are included with a GLR $=1.0$, that is to say that even though the MGs don't contribute power to the system they don't impact the reliability of the system but actually improve it when they can provide a backup to the system. It's important to note that the general reliability of the system depends on the reliability of the microgrids. In this work, reliability data was taken for the microgrids' components of the work done in [62].

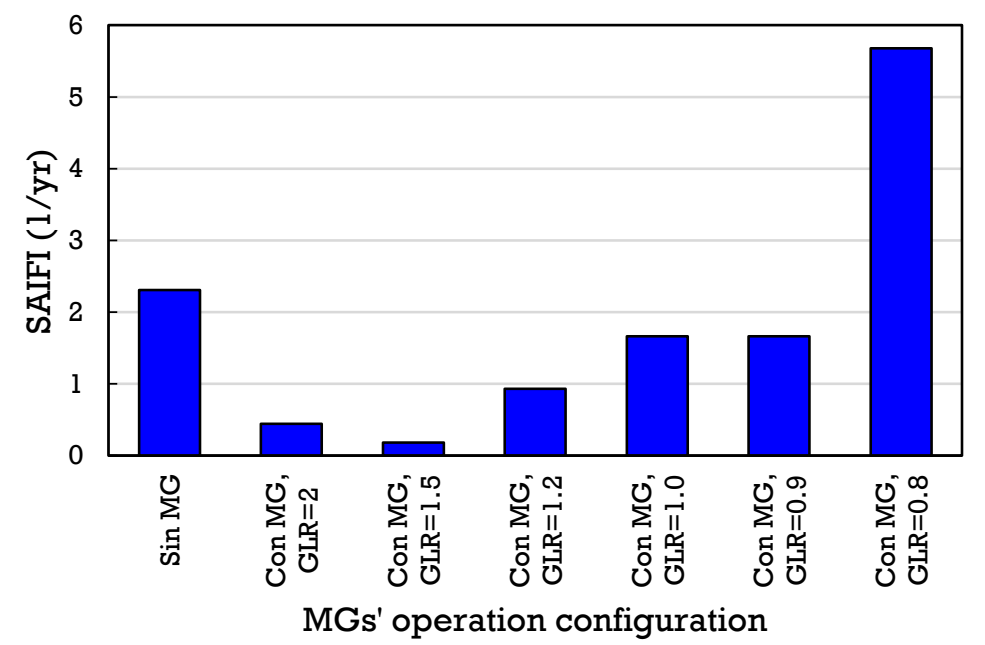

Fig. 8: Behavior of the SAIFI for different values of GLR of the MGs.

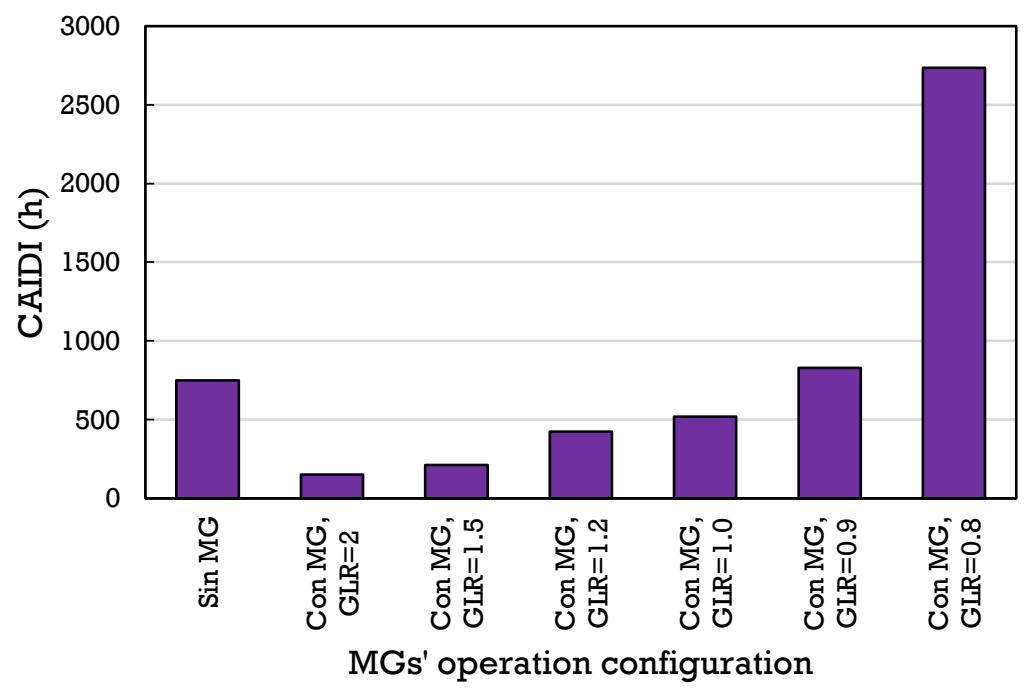

Fig. 9: Behavior of the CAIDI for different values of GLR of the MGs.

The problem of the reliability analyses with multiple microgrids in a distribution system is a high complexity problem. Particularly if is wanted to analyze a way to optimize the reliability of the system, not only are the economical and technical aspects of the systems taken into account, but also the operative conditions of the microgrids, without mentioning that these three aspects are a function of the localization of the DG's resources of the microgrids.

\section{Results: Complex Network Application to Improve the Reliability}

\subsection{Trial Network's Centrality Measures}

Making use of the MATLAB software, the centrality measures for the modified IEEE trial system with 30 nodes were calculated. In Figures 10,11 , and 12 are graphically shown the centrality measures of closeness, betweenness and intermediation considering the chargeability limits of the lines and transformers. The centrality measures of the graph with weights are the ones that 
are taken into account for the selection of plausible nodes for the connection of multiple microgrids in

the considered system.

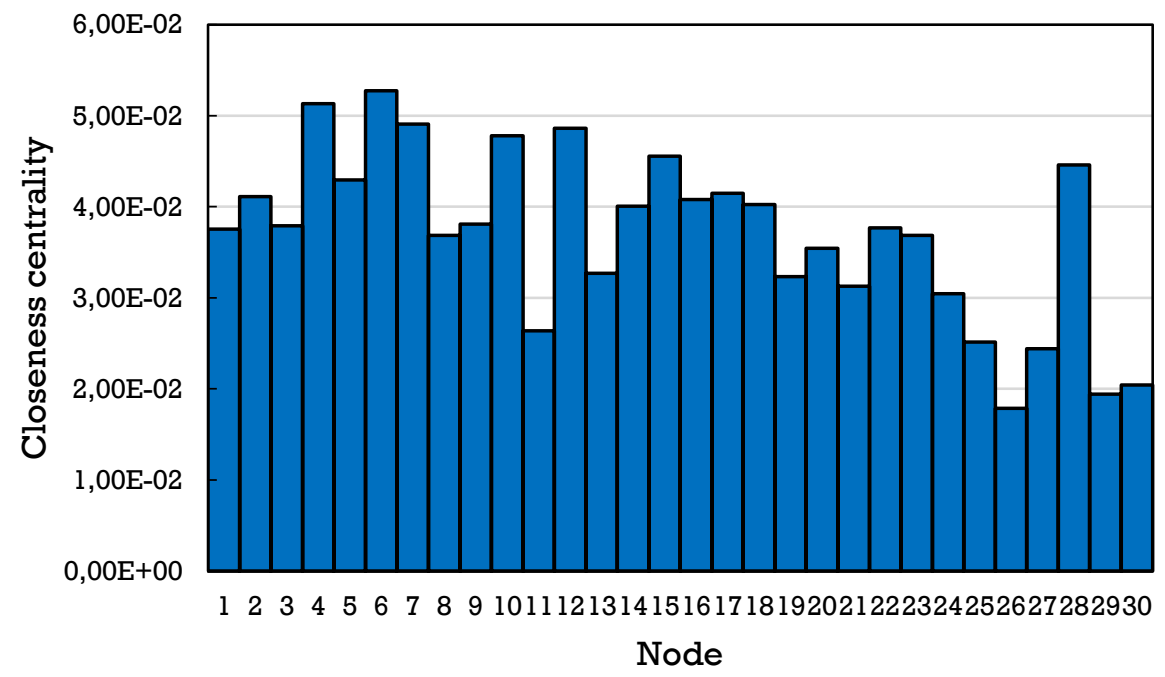

Fig. 10: Closeness centrality for each node.

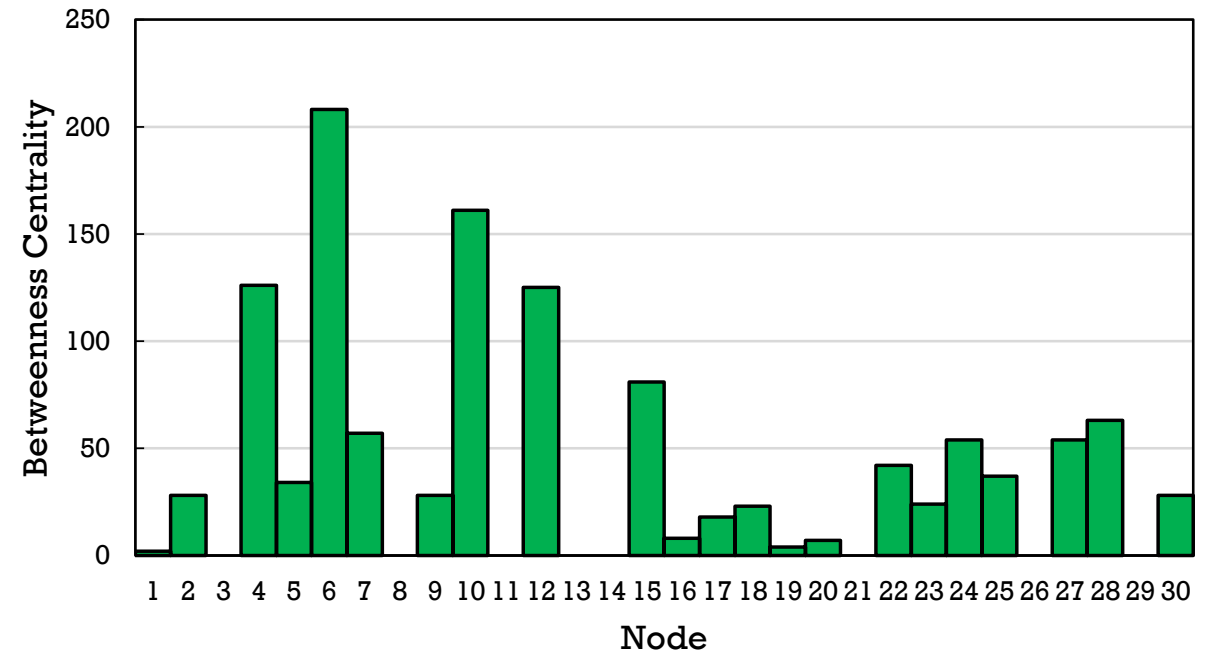

Fig. 11: Betweenness centrality for each node.

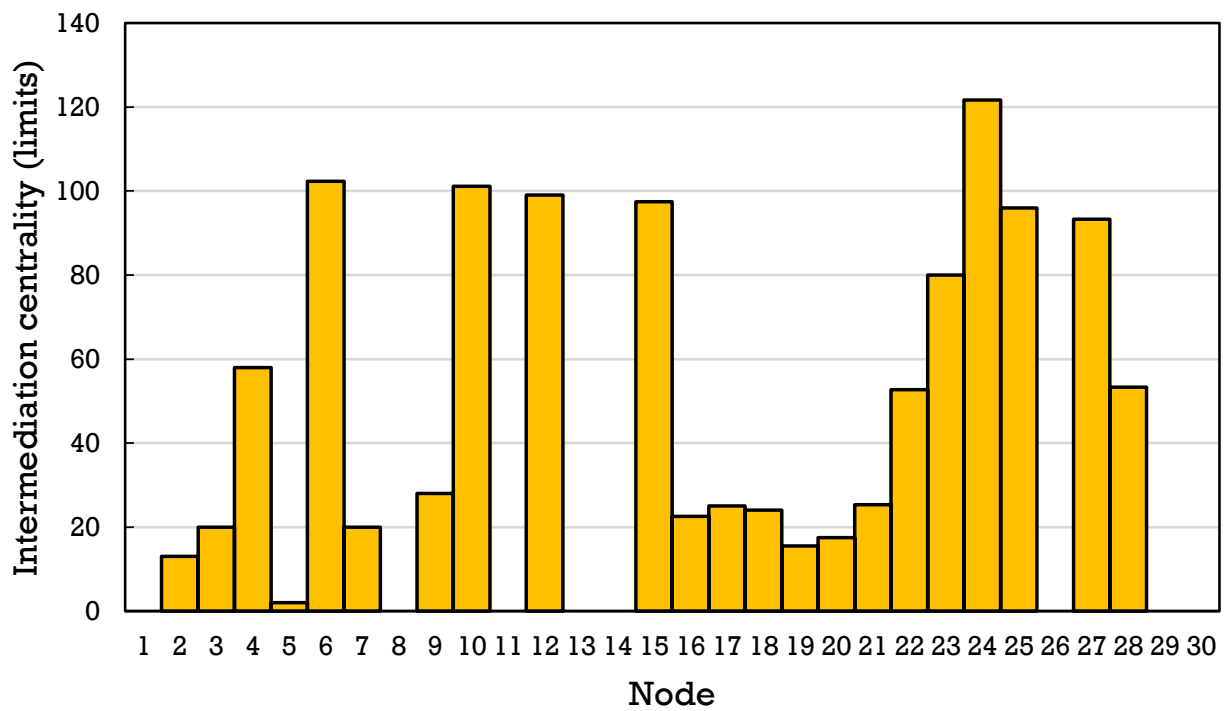

Fig. 12: Intermediation centrality for each node considering the chargeability limits of the elements. 


\subsection{Reliability Analysis based in Complex Network Theory}

Based on the results obtained from the complex network analysis is proposed in this work to select nodes with centrality measures that allow the identification of which are the critical nodes where the microgrids' connection is optimal from a system's reliability point of view.

Selected the connection nodes based on the given measures by the complex network theory, the system's reliability is calculated, without altering any other variables in the network, in a way that the impact in the reliability can be compared. The reliability analysis is made with a GLR of 1.2 for every microgrid.

Localization of the MMG based in closeness centrality.

Taking as reference the first 5 nodes hierarchically classified with the greater closeness centrality indexes, the nodes to assign the microgrids are selected. In Table 3 are shown the 5 nodes with the highest closeness index that considers the graph's weights.

Table 3. Nodes with the highest closeness centrality.

\begin{tabular}{ccc}
\hline \multirow{2}{*}{ Node } & \multicolumn{2}{c}{ Closeness centrality } \\
\cline { 2 - 3 } & Without weights & With weights \\
\hline 6 & 0.015152 & 0.052748 \\
\hline 4 & 0.013333 & 0.051342 \\
\hline 7 & 0.010870 & 0.049101 \\
\hline 12 & 0.012048 & 0.048605 \\
\hline 10 & 0.013889 & 0.047808 \\
\hline
\end{tabular}

Table 4: Results of the reliability calculation for the reconfiguration of the MGs based in closeness.

\begin{tabular}{ccc}
\hline Indicator & Unit & Value \\
\hline SAIFI & $1 / \mathrm{yr}$. & 2.253 \\
\hline SAIDI & $\mathrm{min} / \mathrm{yr}$. & 1298.347 \\
\hline CAIDI & $\mathrm{h}$ & 9.607 \\
\hline $\mathrm{P}$ & $\mathrm{MW} / \mathrm{yr}$. & 880.35 \\
\hline $\mathrm{W}$ & $\mathrm{MWh} / \mathrm{yr}$. & 7977.943 \\
\hline
\end{tabular}

As indicated in Table 4, the SAIDI and the SAIFI increased with respect to the base case analyzed by changing the connection points of the MG based in the closeness centrality measure obtained through the complex network analysis.

Localization of the MMG based in the betweenness centrality
Taking as reference the first 5 nodes hierarchically classified with the greatest betweenness centrality indexes, the nodes are selected to assign the microgrids. For this centrality measure are considered the chargeability limits for the lines and transformers.

Table 5. Nodes with the highest betweenness centrality.

\begin{tabular}{cccc}
\hline \multirow{2}{*}{ Node } & \multicolumn{3}{c}{ Betweenness Centrality } \\
\cline { 2 - 4 } & Without weights & With weights & $\begin{array}{c}\text { With weights considering } \\
\text { limits }\end{array}$ \\
\hline 24 & 56.417 & 54 & 121.67 \\
\hline 6 & 176.58 & 208 & 102.33 \\
\hline 10 & 115.67 & 161 & 101.17 \\
\hline 12 & 87.5 & 125 & 99 \\
\hline 15 & 54 & 81 & 97.5 \\
\hline
\end{tabular}

Table 6. Results of the reliability calculation for the reconfiguration of the MGs based in betweenness.

\begin{tabular}{ccc}
\hline Indicator & Unit & Value \\
\hline SAIFI & $1 / \mathrm{yr}$. & 0.875 \\
\hline SAIDI & $\mathrm{min} / \mathrm{yr}$. & 630.178 \\
\hline CAIDI & $\mathrm{h}$ & 12.003 \\
\hline
\end{tabular}




\begin{tabular}{ccc}
\hline $\mathrm{P}$ & MW/yr. & 392.891 \\
\hline $\mathrm{W}$ & MWh/yr. & 4442.803 \\
\hline
\end{tabular}

As shown in Tables 5 and 6, the SAIDI and SAIFI values for the case in which the system is reconfigured, connecting the MGs in the nodes with highest betweenness are minor than in the base case. Localization of MMG focused in reliability based in complex networks

Taking into account that the reliability indicators calculated for the reconfigurations of the
MGs in the distribution system of 30 nodes show that the quality indicators were improved using the betweenness centrality, but worsen while using the closeness centrality, up next are shown cases in which the nodes with the lowest closeness centrality are taken, also lowest betweenness centrality and a case with random node selection to make the same analysis.

Table 7. Nodes with the lowest closeness centrality.

\begin{tabular}{ccc}
\hline \multirow{2}{*}{ Node } & \multicolumn{2}{c}{ Closeness centrality } \\
\cline { 2 - 3 } & Without Weights & With weights \\
\hline 26 & 0.007874 & 0.017861 \\
\hline 29 & 0.008265 & 0.019412 \\
\hline 30 & 0.008265 & 0.020425 \\
\hline 27 & 0.010638 & 0.024411 \\
\hline 25 & 0.010101 & 0.025163 \\
\hline
\end{tabular}

Table 8. Results of the reliability calculation for the reconfiguration of the MGs based in the lowest closeness.

\begin{tabular}{ccc}
\hline Indicator & Unit & Value \\
\hline SAIFI & $1 / \mathrm{yr}$. & 0.856 \\
\hline SAIDI & $\mathrm{min} / \mathrm{yr}$. & 995.359 \\
\hline CAIDI & $\mathrm{h}$ & 19.388 \\
\hline $\mathrm{P}$ & $\mathrm{MW} / \mathrm{yr}$. & 409.636 \\
\hline $\mathrm{W}$ & $\mathrm{MWh} / \mathrm{yr}$. & 6840.687 \\
\hline
\end{tabular}

As shown in the results of the Tables 7 and 8 , the reliability keeps being greater than the calculated for the base case, however, the quality of service indicators doesn't get too much worse when compared to the selection case of nodes based in the nodes with lowest closeness centrality.
For the case of the lowest betweenness centrality, there are 8 nodes with a value of zero, thus, the five nodes indicated in the Table 9 are selected.

Table 9. Nodes with the lowest betweenness centrality.

\begin{tabular}{cccc}
\hline \multirow{2}{*}{ Node } & \multicolumn{3}{c}{ Betweenness centrality } \\
\cline { 2 - 4 } & Without weights & With weights & $\begin{array}{c}\text { With weights } \\
\text { considering limits }\end{array}$ \\
\hline 14 & 0 & 0 & 0 \\
\hline 26 & 0 & 0 & 0 \\
\hline 13 & 0 & 0 & 0 \\
\hline 11 & 0 & 0 & 0 \\
\hline 29 & 0 & 0 & 0 \\
\hline
\end{tabular}

Table 10. Results of the reliability calculation for the reconfiguration of the MGs based in the lowest closeness.

\begin{tabular}{ccc}
\hline Indicator & Unit & Value \\
\hline SAIFI & $1 / \mathrm{yr}$. & 0.875 \\
\hline SAIDI & $\mathrm{min} / \mathrm{yr}$. & 752.052 \\
\hline CAIDI & $\mathrm{h}$ & 15.773 \\
\hline $\mathrm{P}$ & $\mathrm{MW} / \mathrm{yr}$. & 428.8878 \\
\hline $\mathrm{W}$ & $\mathrm{MWh} / \mathrm{yr}$. & 5562.6351 \\
\hline
\end{tabular}


According to the indicators resulted in the Table 10 is observed that the node selection with the lowest betweenness has a negative impact in the reliability indicators. For this case is noted that some of the nodes with the lowest betweenness correspond to the microgrids connection nodes in the base case. This corresponds that to the microgrids connection nodes in the base case correspond to external nodes of the system, that is to say radial nodes with grade 1 centrality.

\section{Discussion and Conclusion}

With this work is proposed a selection alternative of nodes for connection of multiples microgrids in an electrical distribution system based in the analysis of complex networks. This perspective allows the realization of a quick and efficient selection of the connection nodes of the microgrids that improve the reliability of the system.

The reliability of a distribution network was evaluated including multiple microgrids through the analysis of an electrical network modeled as a complex network. From this perspective, is expected that the nodes with the highest betweenness centrality, a measure provided from the complex network theory, are the nodes that when connected the microgrids they improve the reliability of the whole system more than if they were connected in other nodes. Besides this it can't be concluded that the localization of the microgrids using this unique criterion based in betweenness centrality will be the most optimal localization that minimizes the quality of service indicators (SAIDI and SAIFI) since for this specific case every possible combination of arrays of the available microgrids must be evaluated in every node of the system. Additionally, to perform the localization optimization of the microgrids simulations of Monte Carlo must be made to calculate the reliability and find the minimum of the quality of service indicators through optimization algorithms, such as PSO (Particle Swarm Optimization) or genetical optimization algorithms.

The proposed method in this work for the selection of connection nodes of microgrids can be a useful tool, since to perform an optimization with the fore mentioned methods it requires a high computational consumption and long processing times. The method in which the complex network theory can proportionate a quick, efficient and computationally low costly analysis.

Based on the above, not only it's convenient, but also will be essential that the studies in reliability analyses from the NO in the electrical sector take into account these new technologies of reliability in the planning stage of the distribution systems and take advantage of its potential to fulfill the quality of service regulatory requirements.

It was analyzed the relation that can exist between the closeness and betweenness centrality measures of the system obtained from the complex network analysis with the reliability indicators used in electrical distribution networks (SAIDI, SAIFI) that are measured to evaluate the given service by the NO according to the Colombian regulatory framework. It was observed that the betweenness of closeness does not provide a guide to determine nodes that have an impact in the reliability of the system by connecting multiple microgrids, however, it can exist some relation between the system's betweenness centrality measure since by calculating the reliability of the reorganized system the microgrids in the distribution network based in the nodes with the highest betweenness index low values are obtained in the quality of service indicators, which indicates an improvement in the reliability.

Due to the reliability analysis that involves multiple microgrids in an electrical system involving many variables and it's a very hard problem to confront, for the analysis made in this work several simplifications like modeling the microgrids with the VPP concept were made, assuming a constant GLR for all the microgrids and a power generation and equal consumption for all, as well as a failure rate equal for every microgrid. The generation costs according to the type of DER resource aren't taken into account as well as the charges variability. Considering all the elements to find the connection nodes of the microgrids that optimize the reliability, that is to say they make the quality indicators better (nodes that minimize the SAIDI and SAIFI) is a very complex optimization problem that would require advanced optimization algorithms such as PSO, genetic optimization algorithms, Customer Scattering, etc. These could be applied to the optimization of the reliability calculation through Monte Carlo methods. The study of the complex network analysis can continue with a focus to verifying that it's a quick, simple and very efficient analytic method to find optimal nodes, verifying it with optimization algorithms like the ones mentioned above. The prior is not only for the reliability analyses, but it can be also used in other power systems' analyses.

The complex network theory provides a completely different perspective for the power systems' analysis. The concepts that are applied from the complex network theory to the electrical networks can help us to better comprehend the topology, the characteristics, the behavior and for this particular case the reliability of an electrical distribution network from other focus point. However, in the present the application of the 
complex network theory to the electrical networks is still in a theoretical level and requires greater depth in the researches that lead to being able to apply these concepts and allow the development of ways to improve the reliability, safety, stability and efficiency of the electrical networks.

\section{References:}

[1] A. A. Chowdhury and D. O. Koval, Power Distribution System Reliability. Hoboken, NJ, USA: John Wiley \& Sons, Inc., 2009.

[2] R. Billinton and R. N. Allan, Reliability Evaluation of Power Systems, 2nd ed. Boston, MA: Springer US, 1996.

[3] R. N. Allan, R. Billinton, A. M. Breipohl, and C. H. Grigg, "Bibliography on the application of probability methods in power system reliability evaluation," IEEE Transactions on Power Systems, vol. 14, no. 1, pp. 51-57, 1999, doi: 10.1109/59.744483.

[4] P. Jahangiri and M. Fotuhi-Firuzabad, "Reliability assessment of distribution system with distributed generation," in 2008 IEEE 2nd International Power and Energy Conference, Dec. 2008, pp. 15511556, doi: 10.1109/PECON.2008.4762728.

[5] M. Makandar, C. S. R. Atla, and S. Velamuri, "Reliability assessment of distribution system with renewable Distributed Generation," in 2016 Biennial International Conference on Power and Energy Systems: Towards Sustainable Energy (PESTSE), Jan. 2016, pp. 1-5, doi: 10.1109/PESTSE.2016.7516365.

[6] S. Xin, C. Yan, Z. Xingyou, and W. Chuanzhi, "A novel multi-microgrids system reliability assessment algorithm using parallel computing," in 2017 IEEE Conference on Energy Internet and Energy System Integration (EI2), Nov. 2017, pp. 1-5, doi: 10.1109/EI2.2017.8245363.

[7] M. A. Al-Shehri, Y. Guo, and G. Lei, "A Systematic Review of Reliability Studies of GridConnected Renewable Energy Microgrids," in 2020 International Conference on Electrical, Communication, and Computer Engineering (ICECCE), Jun. 2020, no. June, pp. 1-6, doi: 10.1109/ICECCE49384.2020.9179273.

[8] Z. Bie, P. Zhang, G. Li, B. Hua, M. Meehan, and X. Wang, "Reliability Evaluation of Active Distribution Systems Including Microgrids," IEEE Transactions on Power Systems, vol. 27, no. 4, pp. 2342-2350, Nov. 2012, doi: 10.1109/TPWRS.2012.2202695.

[9] P. M. Costa and M. A. Matos, "Assessing the contribution of microgrids to the reliability of distribution networks," Electric Power Systems Research, vol. 79, no. 2, pp. 382-389, Feb. 2009, doi: 10.1016/j.epsr.2008.07.009.

[10] F. Tooryan and E. R. Collins, "Optimum size and placement of distributed generators in microgrid based on reliability concept," in 2018 IEEE Power and Energy Conference at Illinois (PECI), Feb. 2018, vol. 2018-Janua, pp. 1-6, doi: 10.1109/PECI.2018.8334992.
[11] T. Adefarati and R. C. Bansal, "Reliability assessment of distribution system with the integration of renewable distributed generation," Applied Energy, vol. 185, pp. 158-171, Jan. 2017, doi: 10.1016/j.apenergy.2016.10.087.

[12] Comisión de Regulación de Energía y Gas CREG, Ministerio de Minas y Energía, and Republica de Colombia, Resolución CREG No. 015 de 2018. Colombia, 2018, p. 239.

[13] M. Castaño-Gómez and J. J. García-Rendón, "Análisis de los incentivos económicos en la capacidad instalada de energía solar fotovoltaica en Colombia," Lecturas de Economía, no. 93, pp. 23-64, Jul. 2020, doi: 10.17533/udea.le.n93a338727.

[14] Comision de Regulacion de Energia y Gas CREG; Ministerio de Minas y Energía; Republica de Colombia, Resolución CREG No. 030 de 2018. Colombia, 2018, p. 13.

[15] Comisión de Regulación de Energía y Gas CREG, Ministerio de Minas y Energía, and Republica de Colombia, Resolución CREG No. 038 de 2018. Colombia, 2018.

[16] R. Kaduru and N. Srinivas GONDLALA, "Distribution System Reliability with Distributed Generation Based on Customer Scattering," no. 13, 2015, doi: 10.15598/aeee.v13i2.1025.

[17] Ke Sun, "Complex Networks Theory: A New Method of Research in Power Grid," in 2005 IEEE/PES Transmission \&amp; Distribution Conference \&amp; Exposition: Asia and Pacific, 2005, vol. 2005, pp. 1-6, doi: 10.1109/TDC.2005.1547099.

[18] B. Liu, Z. Li, X. Chen, Y. Huang, and X. Liu, "Recognition and Vulnerability Analysis of Key Nodes in Power Grid Based on Complex Network Centrality," IEEE Transactions on Circuits and Systems II: Express Briefs, vol. 65, no. 3, pp. 346350, 2018, doi: 10.1109/TCSII.2017.2705482.

[19] Y. Nurdin, "Understanding the Cascading Failures in Indonesian Power Grids with Complex Network Theory," in 2019 IEEE International Conference on Cybernetics and Computational Intelligence (CyberneticsCom), Aug. 2019, pp. 50-55, doi: 10.1109/CYBERNETICSCOM.2019.8875659.

[20] A. B. M. Nasiruzzaman and H. R. Pota, "Transient stability assessment of smart power system using complex networks framework," 2011, doi: 10.1109/PES.2011.6038970.

[21] A. Dwivedi, X. Yu, and P. Sokolowski, "Identifying vulnerable lines in a power network using complex network theory," in 2009 IEEE International Symposium on Industrial Electronics, Jul. 2009, no. ISIE, pp. 18-23, doi: 10.1109/ISIE.2009.5214082.

[22] A. T. Alexandridis and P. C. Papageorgiou, "A complex network deployment suitable for modern power distribution analysis at the primary control level," IFAC-PapersOnLine, vol. 50, no. 1, pp. 9186-9191, 2017, doi: 10.1016/j.ifacol.2017.08.1272.

[23] M. Saleh, Y. Esa, N. Onuorah, and A. A. Mohamed, "Optimal microgrids placement in electric distribution systems using complex 
network framework," 2017 6th International Conference on Renewable Energy Research and Applications, ICRERA 2017, vol. 2017-Janua, pp. 1036-1040, 2017, doi: 10.1109/DISTRA.2017.8191215.

[24] EPRI, "Strategic Insights on Security, Quality, Reliability, and Availability," 2005. [Online]. Available:

https://www.epri.com/research/products/00000000 0001008566.

[25] R. Billinton and R. N. Allan, "Power-system reliability in perspective," Electronics and Power, vol. 30, no. 3, p. 231, 1984, doi: 10.1049/ep.1984.0118.

[26] R. Billinton and R. N. Allan, Reliability Evaluation of Engineering Systems, 2nd ed., vol. 43, no. 4. Boston, MA: Springer US, 1992.

[27] M. A. Bucher, M. Vrakopoulou, and G. Andersson, "Probabilistic N-1 security assessment incorporating dynamic line ratings," 2013, doi: 10.1109/PESMG.2013.6672679.

[28] EPRI, "Value Modeling for Reliability of Distribution and Transmission Systems," 2006. [Online]. Available: https://www.epri.com/research/products/00000000 0001012501.

[29] G. C. Loehr, "The 'good' Blackout: The Northeast Power Failure of 9 November 1965 [History]," IEEE Power and Energy Magazine, vol. 15, no. 3. Institute of Electrical and Electronics Engineers Inc., pp. 84-96, May 01, 2017, doi: 10.1109/MPE.2017.2659379.

[30] J. McCalley et al., "Probabilistic security assessment for power system operations," in 2004 IEEE Power Engineering Society General Meeting, 2004, vol. 1, pp. 212-220, doi: 10.1109/pes.2004.1372788.

[31] W. Li and J. Zhou, "Probabilistic reliability assessment of power system operations," Electric Power Components and Systems, vol. 36, no. 10, pp. 1102-1114, Oct. 2008, doi: 10.1080/15325000802046868.

[32] Y. Sun, L. Cheng, X. Ye, J. He, and P. Wang, "Overview of power system operational reliability," in 2010 IEEE 11th International Conference on Probabilistic Methods Applied to Power Systems, PMAPS 2010, 2010, pp. 166-171, doi: 10.1109/PMAPS.2010.5528993.

[33] W. Li, "Evaluating Mean Life of Power System Equipment with Limited End-of-Life Failure Data," IEEE Transactions on Power Systems, vol. 19, no. 1, pp. 236-242, Feb. 2004, doi: 10.1109/TPWRS.2003.821434.

[34] R. Billinton and W. Li, Reliability Assessment of Electric Power Systems Using Monte Carlo Methods, 1st ed. Boston, MA: Springer US, 1994.

[35] R. Billinton and A. Jonnavithula, "Variance reduction techniques for use with sequential Monte Carlo simulation in bulk power system reliability evaluation," in Canadian Conference on Electrical and Computer Engineering, 1996, vol. 1, pp. 416419, doi: 10.1109/ccece.1996.548125.

[36] Y. Wang, C. Guo, Q. Wu, and S. Dong, "Adaptive sequential importance sampling technique for short-term composite power system adequacy evaluation," IET Generation, Transmission and Distribution, vol. 8, no. 4, pp. 730-741, 2014, doi: 10.1049/iet-gtd.2013.0279.

[37] Q. Chen and L. Mili, "Composite power system vulnerability evaluation to cascading failures using importance sampling and antithetic variates," IEEE Transactions on Power Systems, vol. 28, no. 3, pp. 2321-2330, 2013, doi: 10.1109/TPWRS.2013.2238258.

[38] P. Jirutitijaroen and C. Singh, "Comparison of simulation methods for power system reliability indexes and their distributions," IEEE Transactions on Power Systems, vol. 23, no. 2, pp. 486-493, May 2008, doi: 10.1109/TPWRS.2008.919425.

[39] R. H. Lasseter, "Smart distribution: Coupled microgrids," in Proceedings of the IEEE, 2011, vol. 99, no. 6, pp. 1074-1082, doi: 10.1109/JPROC.2011.2114630.

[40] N. D. Hatziargyriou, A. Dimeas, A. G. Tsikalakis, J. A. Pecas Lopes, G. Kariniotakis, and J. Oyarzabal, "Management of microgrids in market environment," in 2005 International Conference on Future Power Systems, 2005, vol. 2005, doi: 10.1109/fps.2005.204225.

[41] P. M. Costa and M. A. Matos, "Economic analysis of microgrids including reliability aspects," 2006, doi: 10.1109/PMAPS.2006.360236.

[42] A. Abdulkarim et al., "Reliability Study of Standalone Hybrid Renewable Energy Microgrids," Iranian Journal of Science and Technology Transactions of Electrical Engineering, vol. 43, no. 1, pp. 411-425, Jul. 2019, doi: 10.1007/s40998018-0119-8.

[43] E. Zio, "Reliability Analysis of Complex Network Systems: Research and Practice in Need," IEEE Transactions on Reliability, vol. 57, no. 3, pp. 1-4, 2008.

[44] J. Huang, Y. Feng, and S. Zhang, "Research of complex system theory application on reliability analysis of network system," Proceedings of 2009 8th International Conference on Reliability, Maintainability and Safety, ICRMS 2009, pp. 1141-1145, 2009, doi: 10.1109/ICRMS.2009.5270062.

[45] M. Newman, Networks: An Introduction. Oxford University Press, 2010.

[46] M. E. J. Newman, "The Structure and Function of Complex Networks," SIAM Review, vol. 45, no. 2, pp. 167-256, Jan. 2003, doi: $10.1137 / \mathrm{S} 003614450342480$.

[47] R. Shields, "Cultural Topology: The Seven Bridges of Königsburg, 1736," Theory, Culture \& Society, vol. 29, no. 4-5, pp. 43-57, Jul. 2012, doi: 10.1177/0263276412451161.

[48] R. Albert and A.-L. Barabási, "Statistical mechanics of complex networks," Reviews of Modern Physics, vol. 74, no. 1, pp. 47-97, Jan. 2002, doi: 10.1103/RevModPhys.74.47.

[49] J. Kim and T. Wilhelm, "What is a complex graph?," Physica A: Statistical Mechanics and its Applications, vol. 387, no. 11, pp. 2637-2652, 2008, doi: 10.1016/j.physa.2008.01.015. 
[50] A. B. M. Nasiruzzaman and H. R. Pota, "Critical node identification of smart power system using complex network framework based centrality approach," 2011, doi: 10.1109/NAPS.2011.6025194.

[51] M. Mesbahi and M. Egerstedt, Graph Theoretic Methods in Multiagent Networks. Princeton University Press, 2010.

[52] L. A. Machuca Moreno, "Análisis de estabilidad transitoria basado en teoría de redes complejas y el fenómeno de percolación," 2017.

[53] G. Zhang, C. Wang, J. Zhang, J. Yang, Y. Zhang, and M. Duan, "Vulnerability assessment of bulk power grid based on complex network theory," 3rd International Conference on Deregulation and Restructuring and Power Technologies, DRPT 2008, no. April, pp. 1554-1558, 2008, doi: 10.1109/DRPT.2008.4523652.

[54] G. Chen, Z. Y. Dong, D. J. Hill, and G. H. Zhang, "An improved model for structural vulnerability analysis of power networks," Physica A: Statistical Mechanics and its Applications, vol. 388, no. 19, pp. 4259-4266, Oct. 2009, doi: 10.1016/j.physa.2009.06.041.

[55] I. Rajasingh, B. Rajan, and I. D. Florence, "Betweeness-centrality of grid networks," in ICCTD 2009 - 2009 International Conference on Computer Technology and Development, 2009, vol. $1, \quad$ pp. 407-410, doi: 10.1109/ICCTD.2009.230.

[56] M. Girvan and M. E. J. Newman, "Community structure in social and biological networks," Proceedings of the National Academy of Sciences of the United States of America, vol. 99, no. 12, pp. 7821-7826, Dec. 2001, doi: 10.1073/pnas.122653799.

[57] L. C. Freeman, "Centrality in social networks conceptual clarification," Social Networks, vol. 1, no. 3, pp. 215-239, Jan. 1978, doi: 10.1016/03788733(78)90021-7.

[58] R. Christie, "Power Systems Test Case Archive 30 Bus Power Flow Test Case," 1993. http://labs.ece.uw.edu/pstca/pf30/pg_tca30bus.htm (accessed Jan. 23, 2021).

[59] The Grainger College of Engineering and University of Illinois at Urbana-Champaign, "Illinois Center for a Smarter Electric Grid (ICSEG). IEEE 30-Bus System," 2013. [Online]. Available: https://icseg.iti.illinois.edu/ieee-30-bussystem/.

[60] "IEEE Recommended Practice for the Design of Reliable Industrial and Commercial Power Systems," IEEE Std 493-2007 (Revision of IEEE Std 493-1997), pp. 1-383, 2007, doi: 10.1109/IEEESTD.2007.380668.

[61] Comision de Regulacion de Energia y Gas CREG; Ministerio de Minas y Energía; Republica de Colombia, Resolución CREG No. 025 de 1995. Colombia, 1955.

[62] S. Peyghami, M. Fotuhi-Firuzabad, and F. Blaabjerg, "Reliability Evaluation in Microgrids With Non-Exponential Failure Rates of Power Units,” IEEE Systems Journal, vol. 14, no. 2, pp.
2861-2872, Jun. 2020, doi: 10.1109/JSYST.2019.2947663.

[63] Z. Bie, P. Zhang, G. Li, B. Hua, M. Meehan, and $\mathrm{X}$. Wang, "Reliability evaluation of active distribution systems including microgrids," IEEE Transactions on Power Systems, vol. 27, no. 4, pp. 2342-2350, 2012, doi: 10.1109/TPWRS.2012.2202695.

\section{Creative Commons Attribution License 4.0 (Attribution 4.0 International, CC BY 4.0)}

This article is published under the terms of the Creative Commons Attribution License 4.0 https://creativecommons.org/licenses/by/4.0/deed.en US 